\title{
¿Qué recursos didácticos conocen y usan nuestros alumnos?
}

\section{D. de Andrés Martínez ${ }^{\mathrm{a}}$, E. Antonino Daviu ${ }^{\mathrm{b}}$, C. Gisbert Doménech ${ }^{\mathrm{c}}$ J.C. Ruiz García ${ }^{\mathrm{d}}$, A. Palomares Chust ${ }^{\mathrm{e}}$, A. Peña Cerdán ${ }^{\mathrm{f}}$}

${ }^{a}$ Universitat Politècnica de València, Dpto. de Informática de Sistemas y Computadores, ddandres@disca.upv.es, ${ }^{b}$ Universitat Politècnica de València, Dpto .de Comunicaciones, evanda@upvnet.upv.es, ' Universitat Politècnica de València, Dpto. de Biotecnología, cgisbert@btc.upv.es, ${ }^{d}$ Universitat Politècnica de València, Dpto. de Informática de Sistemas y Computadores, jcruizg@disca.upv.es, ${ }^{e}$ Universitat Politècnica de València, Dpto. de Sistemas Informáticos y Computación, apalomares@dsic.upv.es, ${ }^{f}$ Universitat Politècnica de València, Dpto. de Proyectos Arquitectónicos, anpecer2@pra.upv.es

\begin{abstract}
The Universitat Politècnica de València (UPV) is promoting for some years now, the design, elaboration and use of different multimedia learning materials, such as videos, screencasst and slide-based presentations. The goal is to support and encourage the out-of-class work of students. Although a lot of effort has been placed so far in improving the content of such materials, few research has been carried out on the use of such materials and its actual impact on the students' learning process and academic performance. This research wants to determine the degree of awareness and relevance that students provide to the existence and use of this type of multimedia materials. The study will be focused on three different areas of expertise, such as those defined by the engineering, architecture and tourism domains. It will compare the importance for students of the different available materials at UPV with respect to other multimedia resources available on Internet.
\end{abstract}

Keywords: Multimedia resources, learning, out-of-class work, Riunet

\begin{abstract}
Resumen
La Universitat Politècnica de València (UPV) lleva ya unos años promoviendo e incentivando el diseño, elaboración y uso de distintos tipos de materiales multimedia, como videos explicativos, screencasts $y$ presentaciones, con el objetivo de dinamizar el trabajo del alumno fuera del aula. Aunque muchos trabajos se han centrado en los últimos años en desarrollar mejores contenidos multimedia, poca investigación se ha llevado a cabo hasta la fecha en el estudio del uso que los alumnos hacen de dicho material y en el impacto que el mismo tiene, en un primer momento, en su proceso de aprendizaje, y finalmente, en sus resultados académicos. El objetivo de esta comunicación es determinar el grado de conocimiento y uso que dan los alumnos al material multimedia del que disponen. Este estudio se efectuará en distintas áreas de conocimiento en el ámbito de la ingeniería, la arquitectura y el turismo. Se pretenden identificar el grado de conocimiento que tienen los alumnos de los recursos multimedia de la UPV y el grado de
\end{abstract}


uso que hacen de este material y de otros materiales multimedia disponibles en Internet.

Palabras clave: Recursos multimedia, aprendizaje, trabajo no-presencial, Riunet

\section{Introducción}

La introducción de recursos multimedia en la educación permite a los profesores mejorar sus métodos de enseñanza y evaluación a través de una serie de herramientas que combinan texto, gráficos, audio y vídeo (Barra, 2014), transformando las clases del presente en entornos de aprendizaje ricos, interactivos y centrados en el estudiante (Mahajan, 2012). En el año 2007 se inició en la Universitat Politècnica de València (UPV) el programa "Docencia en Red", con el fin de incentivar al profesorado en la elaboración de materiales multimedia educativos con el fin de incluirlos en Riunet, el repositorio institucional de la UPV (Universitat Politècnica de València, 2013). Entre los materiales que se elaboran en el marco de este programa se incluyen los objetos de aprendizaje mostrados en la Fig. 1. Todos los recursos elaborados se depositan en RiuNet donde se publican en abierto. El programa sin duda ha resultado exitoso puesto que el número de materiales se incrementa anualmente a muy buen ritmo (actualmente, a 29 de marzo de 2017, el repositorio cuenta con un total de 6425 objetos de aprendizaje) y contribuye también a la difusión de la UPV al resto del mundo. Como ejemplo, en el curso 2014-15 se elaboraron en la UPV dentro de este programa 665 materiales educativos realizados por un total de 140 participantes.

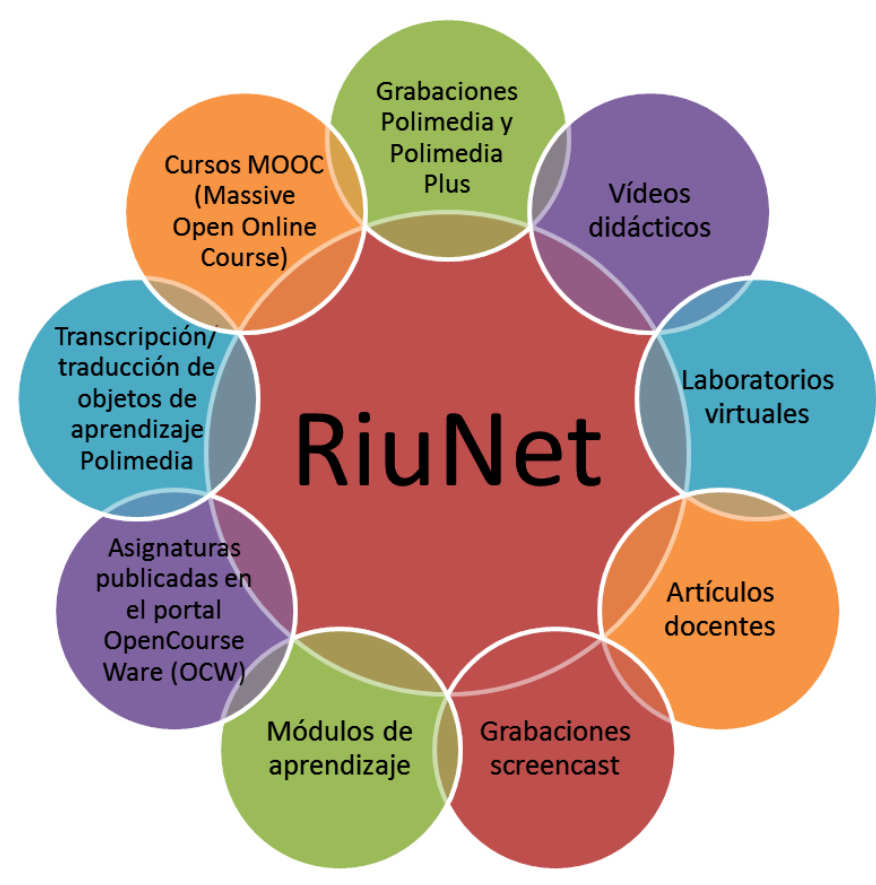

Fig. 1 Objetos de aprendizaje recogidos en el repositorio institucional de la UPV (RiuNet).

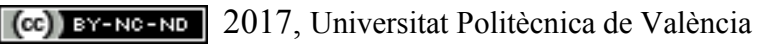


Sin embargo, la utilización de objetos de aprendizaje como recurso didáctico requiere de nuevos enfoques en su diseño, en la metodología docente y en las estrategias de aprendizaje del alumno (Instituto de Ciencias de la Educacion - Área de Sistemas de Información y Comunicaciones, 2008). Como indica Androulla Vassiliou, Comisaria de Educación, Cultura, Multilingüismo y Juventud, "las tecnologías de la información y la comunicación están evolucionando muy rápidamente y las cuestiones relacionadas con su uso en el ámbito educativo resultan cada vez más complejas. Si las herramientas TIC han de convertirse en instrumentos efectivos e integrales en la educación, el seguimiento y la evaluación de este proceso son indispensables" (Agencia Ejecutiva en el Ámbito Educativo, Audiovisual y Cultural, 2011).

En la mayor parte de trabajos relacionados con recursos didácticos, hasta la fecha, dicha evaluación se ha centrado en la satisfacción del alumnado con los objetos de aprendizaje generados para una asignatura concreta (Moltó, 2012), o con la nueva metodología docente soportada por estos recursos didácticos (Jordán, 2014). Sin embargo, no se disponen de datos relativos a las estrategias de aprendizaje desplegadas por el estudiante, que le deben llevar a desarrollar diversas competencias de gestión de la información, organización y planificación, entre otras. Como ejemplo de los aspectos que deberían evaluarse, más allá del impacto particular de la utilización de objetos de aprendizaje en una asignatura determinada, cabría preguntarse cuál es el grado de conocimiento que tienen nuestros alumnos acerca de los recursos multimedia disponibles en el repositorio institucional, si los utilizan para su aprendizaje de forma autónoma o lo hacen únicamente bajo indicación el profesor, si conocen los diferentes tipos de materiales que pueden encontrar en este repositorio y cómo localizarlos, si los valoran adecuadamente, o si les resultan realmente útiles.

Las respuestas a estas cuestiones y la comparación de resultados entre distintas áreas de conocimiento nos van a permitir, además, conocer la conveniencia de elaborar o no otros materiales complementarios, de realizar una mayor difusión entre los alumnos de los recursos disponibles, de aumentar la concienciación del interés que representan estos objetos de aprendizaje para su formación autónoma, determinar aquellos que han resultado más exitosos en cada área de conocimiento y qué aspectos clave han contribuido a su éxito.

Conocedores y participantes de este programa de la UPV "Docencia en Red", en el Equipo de Innovación y Calidad Educativa TASCA (Tools And Strategies for Competences Assessment) nos planteamos una serie de preguntas a las que se pretende dar respuesta en este trabajo, que se enmarca dentro del Proyecto de Innovación y Mejora Educativa PIME/2016/A/029 titulado "Uso e impacto en la docencia universitaria del material didáctico multimedia-UPV". En la Fig. 2 se muestran las preguntas a las que se pretende dar respuesta en este trabajo, en relación al uso e impacto del material didáctico multimedia de la UPV en la docencia universitaria. 
¿Conocen nuestros alumnos los recursos multimedia del repositorio?

¿Utilizan nuestros alumnos dicho recursos?

¿Lo hacen únicamente en el caso de indicarlo el profesor?

¿Conocen realmente todo lo que pueden encontrar en este repositorio?

¿Saben valorarlo?

¿En el caso de utilizar estos materiales, les resultan útiles?

Fig. 2 Preguntas plateadas en el trabajo sobre el uso e impacto del material didáctico multimedia de la UPV en la docencia universitaria.

Las respuestas a estas cuestiones y la comparación de resultados entre áreas de conocimiento nos van a permitir, además, conocer la conveniencia de elaborar o no otros materiales complementarios, de dar más difusión entre los alumnos a los recursos de que disponen, de aumentar la concienciación del interés que representan estos objetos docentes para su formación autónoma, determinar aquellos que han resultado más exitosos en cada área de conocimiento, etc.

En estos momentos nos encontramos en una situación en la que los alumnos que llegan a la Universidad han crecido rodeados de información y tecnología, están acostumbradas a la velocidad y les gusta la innovación (Prensky, 2010). Por otra parte, y en nuestra opinión, están demasiado acostumbrados a que les den todo hecho, por lo que buscan la respuesta fácil sin contrastar, sin buscar información, porque todo tiene que estar en las presentaciones que los profesores depositan en las plataformas docentes (PoliformaT, instancia de SAKAI, en el caso de la UPV). Esto conlleva también a que su atención y concentración en clase sea bastante reducida.

Con esta situación pensamos que es interesante analizar si la inversión que se realiza en la elaboración de todos estos materiales multimedia y que supone un esfuerzo por parte del profesorado se utiliza correctamente. Para que el proceso de enseñanza-aprendizaje sea satisfactorio, los alumnos deben conocer lo que tienen a su disposición, saber valorarlo y darle uso. Los materiales didácticos son herramientas de gran interés para el fomento del aprendizaje autónomo, y los estudios que queremos realizar nos indicarán si están o no infrautilizados. Ayudará también a que el alumno valore la importancia de aprender a aprender para que su proceso de aprendizaje sea lo más eficaz posible, ofreciendo los mejores resultados posibles en base a las tareas encomendadas y al tiempo disponible para hacerles frente. El Espacio Europeo de Educación Superior reconoce este hecho y valora el trabajo del alumno a través de créditos de tipo ECTS, que cuantifican tanto el trabajo presencial del alumno, como el trabajo no presencial que éste debe realizar de forma autónoma. Típicamente la relación entre trabajo presencial y no presencial es de 1:1,5, lo que significa que el trabajo fuera de clase supera al realizado en clase, por lo que conseguir que ese tiempo sea provechoso para el alumno resulta de la máxima importancia. La

(cc) EY-NC-ND 2017, Universitat Politècnica de València

Congreso IN-RED (2017) 
utilización de todos los materiales elaborados en nuestra Universidad y otros que puedan consultar son de gran importancia para conseguir un buen aprendizaje fuera del aula.

Para facilitar el análisis de la situación actual, el resto del artículo se articula de acuerdo a la siguiente estructura. La Sección 2 presenta los diferentes hitos planteados en el proyecto. La Sección 3 detalla el contexto y los aspectos técnicos de la innovación realizada. La Sección 4 analiza los resultados obtenidos y la Sección 5 resume las contribuciones realizadas y el trabajo futuro a abordar.

\section{Objetivos}

El objetivo principal del trabajo es contestar a las preguntas planteadas en la Fig.2, para así conocer el uso que se le está dando por parte del alumnado de los recursos multimedia que se elaboran en la UPV y su opinión al respecto. Estos recursos, depositados en el repositorio Riunet de la UPV, son sin duda herramientas de gran valor para el aprendizaje de los alumnos. Los profesores están contribuyendo a que cada curso el número de recursos aumente y evaluar su utilización por parte del alumnado pensamos que es de gran interés.

Los hitos que se han planteado son:

- Medir el grado de conocimiento que los alumnos tienen de los recursos multimedia UPV.

- Evaluar la utilización de los recursos en el contexto de las asignaturas que imparten los profesores del grupo TASCA por parte de nuestro alumnado.

- Evaluar la utilización de recursos multimedia de los alumnos en otras asignaturas.

- Conocer el grado de satisfacción de los alumnos con los recursos utilizados y sus propuestas de mejora.

- Comparar resultados y definir el planteamiento de nuevas actuaciones relacionadas: difusión, preparación de nuevos materiales, etc.

En esta comunicación se muestran los primeros datos recopilados en diferentes asignaturas y titulaciones de la Universitat Politècnica de València, durante el perimer semestre del curso 2016/2017. Los datos has sido recogidos a través de un cuestionario web realizado a los alumnos y se han extraído las primeras conclusiones.

\section{Desarrollo de la innovación}

Con el fin de conseguir los objetivos planteados, se ha elaborado un cuestionario a través de Google Forms para obtener información de los materiales didácticos multimedia conocidos y utilizados por alumnos en 6 asignaturas (Arquitecturas Telemáticas, Informática de Gestión, Arquitectura e ingeniería de computadores, Cultivo in vitro y transformación genética de plantas, Mejora Genética para la producción vegetal, Sistemas Informáticos en Red Seguros y Confiables) de 6 titulaciones diferentes (Grado en Ingeniería de Tecnologías y Servicios de Telecomunicación, Grado en Turismo, Grado en Ingeniería Informática, Grado en Biotecnología, Grado en Ingeniería Agroalimentaria y del Medio Rural, y Máster Universitario en Ingeniería de Computadores y Redes, respectivamente).

El cuestionario básico elaborado se muestra en la Fig. 3. 


\section{Materiales didácticos multimedia}

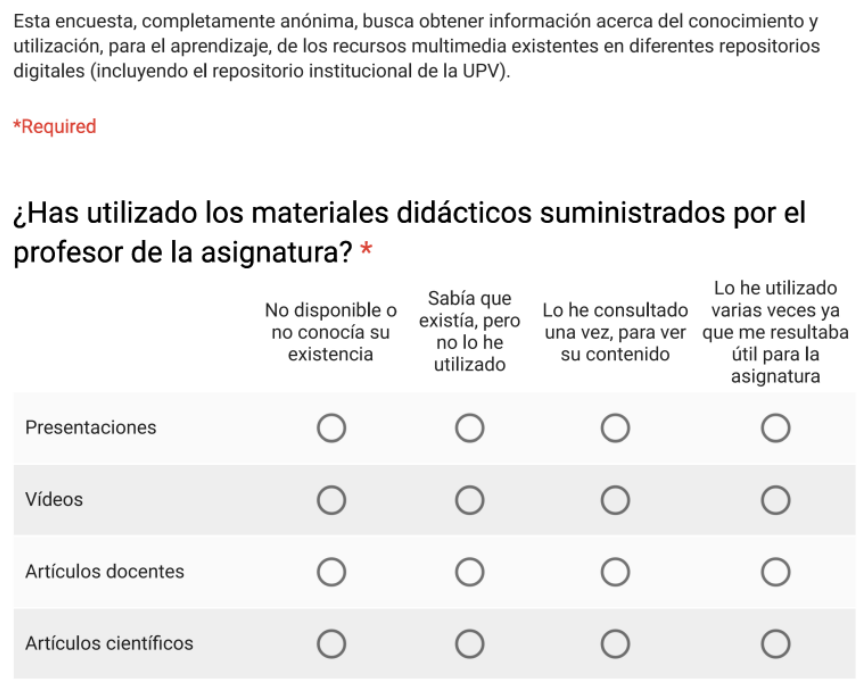

¿Conoces y/o utilizas para el aprendizaje los siguientes repositorios de recusos didácticos multimedia? *

\begin{tabular}{|c|c|c|c|c|}
\hline & $\begin{array}{c}\text { Es la } \\
\text { primera } \\
\text { vez que lo } \\
\text { oigo }\end{array}$ & $\begin{array}{c}\text { Lo } \\
\text { conozco, } \\
\text { pero no lo } \\
\text { he } \\
\text { utilizado } \\
\text { nunca }\end{array}$ & $\begin{array}{l}\text { Lo he } \\
\text { utilizado } \\
\text { alguna vez, } \\
\text { de forma } \\
\text { esporádica }\end{array}$ & $\begin{array}{l}\text { Lo he } \\
\text { utilizado en } \\
\text { diversas } \\
\text { ocasiones y } \\
\text { asignaturas }\end{array}$ \\
\hline \multicolumn{5}{|l|}{ RiuNet (https://riunet.upv.es/) } \\
\hline \multicolumn{5}{|l|}{ mediaUPV (https://media.upv.es/) } \\
\hline \multicolumn{5}{|l|}{ PoliBuscador (http://polibuscador.upv.es) } \\
\hline \multicolumn{5}{|l|}{$\begin{array}{l}\text { poli[OCW] } \\
\text { (http://www.upv.es/contenidos/OCW/) }\end{array}$} \\
\hline \multicolumn{5}{|l|}{ UPV[X] (http://edx.upv.es/) } \\
\hline \multicolumn{5}{|l|}{ Coursera (https://www.coursera.org/) } \\
\hline \multicolumn{5}{|l|}{ edX (https://www.edx.org/) } \\
\hline \multicolumn{5}{|l|}{$\begin{array}{l}\text { Open Education Consortium } \\
\text { (http://www.oeconsortium.org/) }\end{array}$} \\
\hline YouTube (https://www.youtube.com/) & & & & \\
\hline
\end{tabular}

Indica otros repositorios de recursos didácticos multimedia que conozcas

Your answer

Fig. 3 Cuestionario sobre el conocimiento y uso, por parte de los alumnos, del material didáctico multimedia.

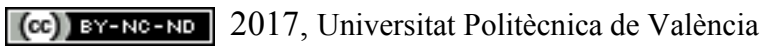


Para aprovechar toda la potencia de Google Forms, y reducir la complejidad del cuestionario, se ha creado una plantilla básica que se ha replicado para cada asignatura. Las respuestas proporcionadas por los alumnos se recogen en una hoja de cálculo diferente para cada cuestionario (asignatura), por lo que es muy cómodo para realizar un análisis de estos datos en base a las asignaturas particulares, los cursos en los que se imparten, o en conjunto.

\section{Resultados}

Hay que destacar que, por el momento, únicamente disponemos de los datos recogidos en asignaturas impartidas durante el primer cuatrimestre del curso 2016-2017, por lo que deberemos esperar al final del presente curso para poder obtener datos del segundo cuatrimestre $\mathrm{y}$, por tanto, más representativos del conjunto de alumnos de la UPV. Sin embargo, el análisis de los datos datos recogidos permite obtener una primera idea acerca del conocimiento y uso de los repositorios de material multimedia existentes.

En primer lugar, cabe indicar que un total de 101 alumnos respondió a la encuesta en las 6 asignaturas consideradas, siendo la asignatura de máster la que menor participación registró (únicamente 3 alumnos) y la del Grado en Turismo la que mayor número de alumnos participó (42). La distribución de alumnos por asignatura se muestra en la Fig. 4.

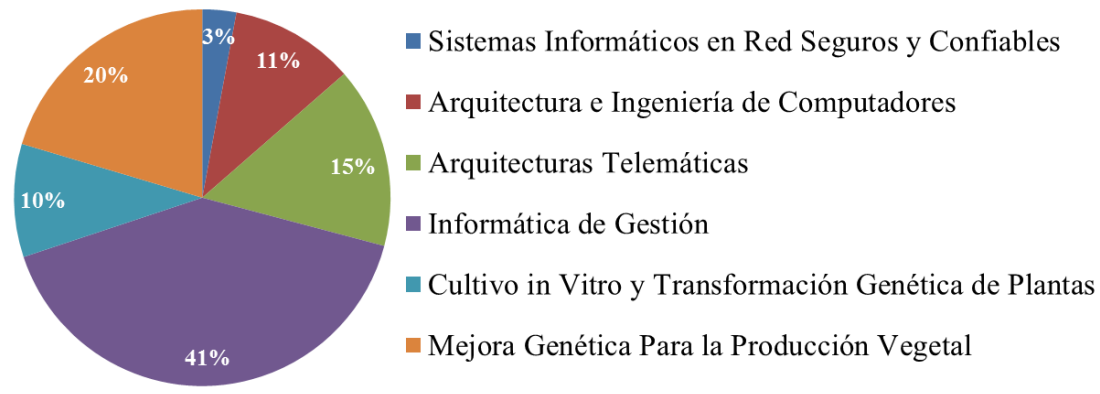

Fig. 4 Distribución en asignaturas de los alumnos que participaron en el cuestionario.

En cuanto a la distribución de alumnos por curso (ver Fig. 5), cabe destacar que para cada curso disponemos de información relativa a una única asignatura (dos en el caso de cuarto curso), debido a la distribución de la carga docente del conjunto de profesores participante en este estudio. Asimismo, la participación de los alumnos de máster ha sido testimonial (un 3\% del total, correspondiente únicamente a 3 alumnos), por lo que los datos obtenidos en este nivel no pueden considerarse representativos del conjunto de estudiantes de máster, aunque se tendrán en cuenta para el análisis global de los datos. 


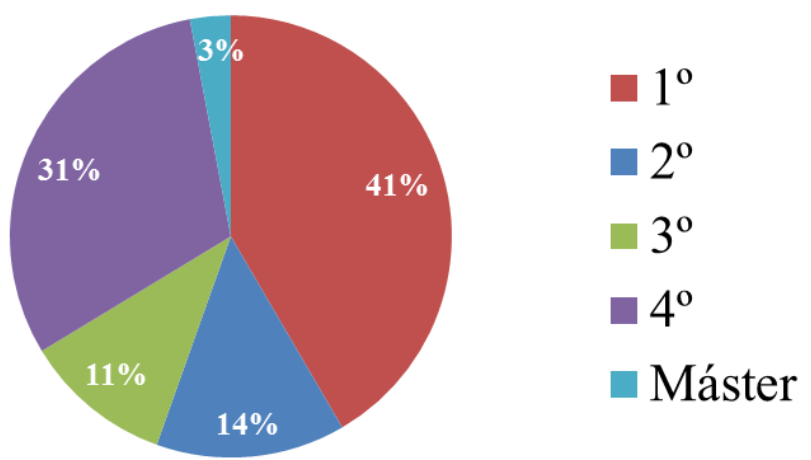

Fig. 5 Distribución en cursos de los alumnos que participaron en el cuestionario.

A continuación se analizarán los datos obtenidos en las dos cuestiones planteadas desde la perspectiva del curso del alumnado participante y en su conjunto.

\subsection{Utilización de los materiales didácticos suministrados por el profesor}

Esta sección analiza el uso que los alumnos declaran haber hecho de los materiales proporcionados por el profesor, principalmente a través del LMS de la UPV (PoliformaT). La distribución del uso que hacen los alumnos de este material, agrupados por cursos, se muestra en la Fig. 6.

En primer lugar, cabe destacar el uso masivo que se hace de las presentaciones (transparencias, diapositivas) que suele utilizar el profesor durante sus clases y que deja disponibles para el alumnado. Cerca del $90 \%$ de los alumnos hacen uso prácticamente exclusivo de este material, un $50 \%$ hace uso de los vídeos indicados, y prácticamente ningún alumno, salvo honrosas excepciones, consulta otros materiales suministrado. Es especialmente preocupante que alrededor del $30 \%$ de los alumnos declare ni siquiera conocer la existencia de este material.

Esta dependencia de las presentaciones del profesor se relaja algo en cursos superiores (alrededor de un $70 \%$ de los alumnos la siguen consultando de manera asidua), ya que los alumnos alcanzan mayor independencia (aprenden a aprender) y buscan consultar nuevos materiales para entender y dominar las materias cursadas. Al menos la mitad del alumnado en estos cursos declara consultar, aunque no demanera asidua, el material complementario en forma de artículos docentes o de investigación. Es significativo el gran uso que se hace de los vídeos, particularmente en cuatro curso, posiblemente debido al carácter práctico de las asignaturas ofertadas como optativas en estos cursos y que suelen proporcionar material audiovisual para reforzar el dominio de las herramientas utilizadas a nivel profesional.

(c)) BY-NC-ND 2017, Universitat Politècnica de València 


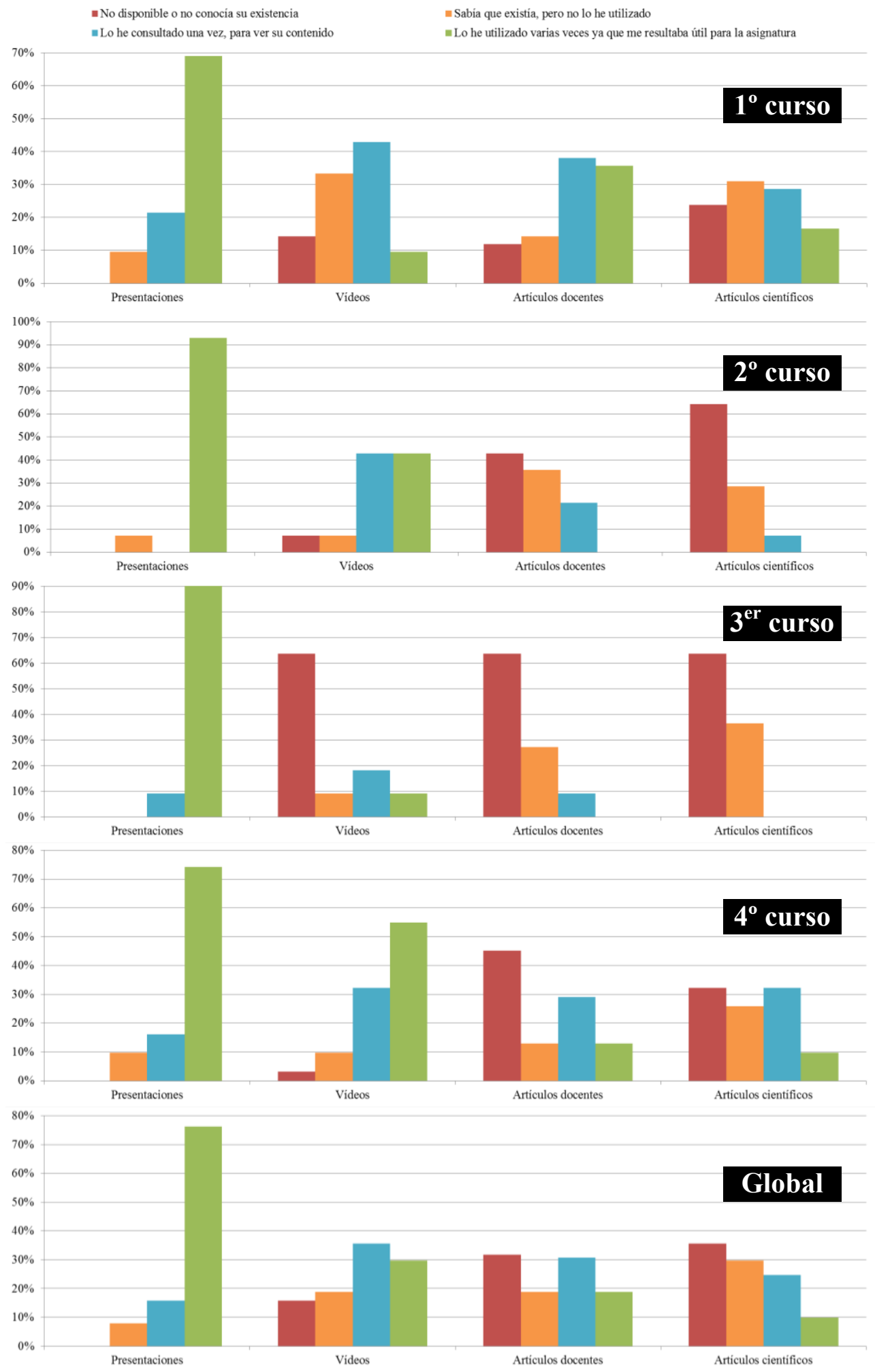

Fig. 6 Conocimiento y utilización por parte del alumno, agrupado por cursos, del material proporcionado por el profesor (presentaciones, vídeos, artículos docentes y artículos científicos). 


\subsection{Conocimiento y utilización de repositorios de materiales didácticos}

En esta sección se analiza el conocimiento y utilización que declaran hacer o haber hecho los alumnos de los diversos repositorios de materiales didácticos existentes, tanto los institucionales de la UPV (Riunet, por ejemplo) como otros de carácter abierto. La distribución del conocimiento y uso declarado, agrupado por cursos, se muestra en la Fig. 7.

En primer lugar, cabe destacar el éxito abrumador de YouTube como canal de distribución de vídeos, donde los alumnos buscan información relativa a las asignaturas que están cursando. En cursos superiores, aunque el porcentaje de alumnos que lo utilizan como vehículo principal para la búqueda de material complementario se reduce ligeramente, continúa situándose por encima del $70 \%$.

En general, los diversos repositorios y buscadores de materiales didácticos son unos grandes desconocidos para los alumnos de la UPV. A nivel institucional, al menos el 50\% de los alumnos ha utilizado alguna vez PoliBuscador, para la localización de todo tipo de recursos didácticos, y mediaUPV, para la localización y visualización de recusos didácticos en formato vídeo. Curiosamente el repositorio institucional de recursos, Riunet, está completamente infrautilizado hasta que los alumnos alcanzan cursos superiores y tratan de localizar recursos adicionales a los proporcionados directamente por el profesorado.

Las grandes plataformas que ofrecen MOOC (Massive Open Online Courses) tampoco tienen gran aceptación entre nuestro alumnado. Únicamente UPV[X], la plataforma MOOC institucional, presenta cierto reconocimiento por parte del alumnado (alrededor del $30 \%$ lo ha utilizado alguna vez).

Hay que remarcar que los resultados obtenidos en segundo curso, que indican un mayor conocimiento de los repositorios existentes que en niveles superiores, se corresponden con una única asignatura. Será preciso analizar los datos recogidos al finalizar el segundo cuatrimestre del curso para poder hacer determinar si estos mejores resultados se deben (muy posiblemente) a la labor realizada por el profesorado en dicha asignatura (o asignatura previas), o a una evolución de la tipología y método de estudio del alumnado. 


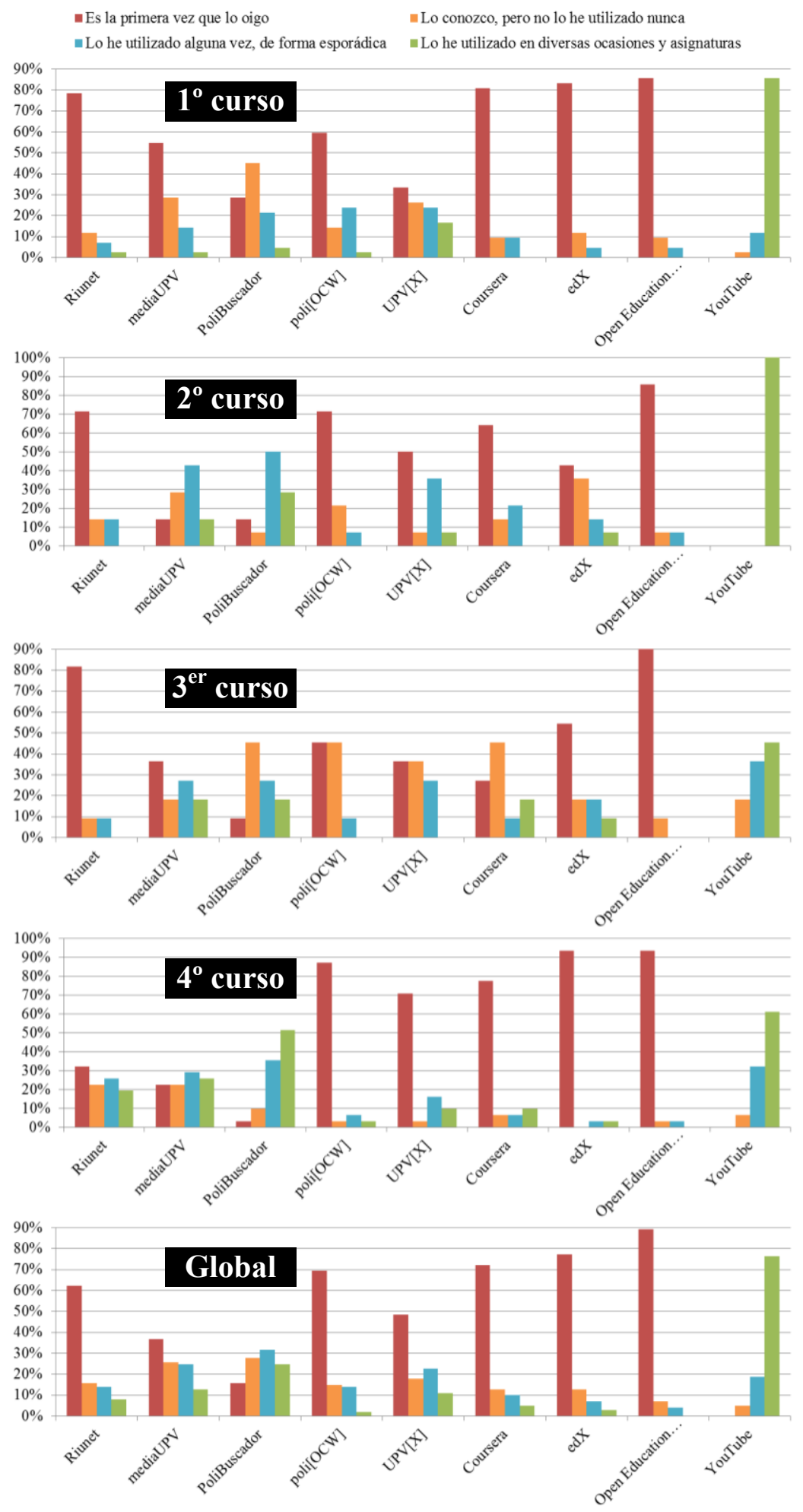

Fig. 7 Conocimiento y utilización por parte del alumno, agrupado por cursos, de repositorios de materiales didácticos.

(cc)) BY-NC-ND 2017, Universitat Politècnica de València 


\section{Conclusiones}

A pesar de encontrarnos en la fases iniciales del proyecto y, por tanto, disponer únicamente de resultados parciales correspondientes a un único cuatrimestre del curso académico, el análisis de los datos recogidos muestra ciertas tendencias generales y, en general, preocupantes.

Las universidades en general, y la UPV en particular, están realizando un esfuerzo enorme para poner a disposición del alumnado multitud de recursos didácticos a través de diferentes repositorios y de herramientas para localizar los recursos deseados en los mismos. Sin embargo, esta iniciativa puede no estar dando los frutos deseados, ya que el conocimiento que tienen los alumnos de estos recursos es mínimo, y únicamente en últimos cursos hacen cierto uso de los materiales disponibles.

Es por tanto, labor de los profesores involucrados en las diferentes asignaturas, promover la utilización de los buscadores y repositorios entre los alumnos para que desarrollen su autonomía y las competencias de búsqueda y gestión de la información y de aprender a aprender. De hecho, la mayor parte de los alumnos únicamente busca información en las presentaciones que el profesor deja disponibles en el LMS o, para información de tipo práctico, a través de YouTube. Sería necesario realizar actividades en las que los alumnos tuvieran que localizar la información complementaria necesaria a través de estos repositorios, para favorecer su conocimiento y uso, y el desarrollo de las habilidades correspondientes por parte del alumnado.

El trabajo futuro a desplegar durante la duración de este proyecto consta de las siguientes fases:

- Recogida y análisis de los datos de las asignaturas impartidas durante el segundo cuatrimestre del curso actual.

- Verificación de los datos recogidos en las encuestas a través de la comprobación de las estadísticas de uso, suministradas por el LMS de la UPV, de los diferentes recursos proporcionados en cada asignatura.

- Comprobación de las estadísticas de uso, proporcionadas por Riunet, acerca de los diferentes recursos disponibles en el repositorio institucional de la UPV. Así, podremos determinar el uso que se hace de estos recursos, distinguiendo aquellos accesos que se realizan localmente (Valencia, España) de los que se realizan a nivel internacional (otros países). Por ejemplo, muchos accesos al material multimedia en español se realiza desde países latinoamericanos, mientras que el alumnado propio de la UPV tiende a ignorar este material. Si esta hipótesis se corrobora, deberán definirse políticas para fomentar el uso interno de estos materiales.

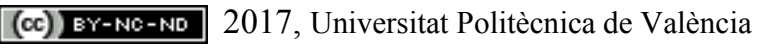




\section{Referencias}

AGENCIA EJECUTIVA EN EL ÁMBITO EDUCATIVO, AUDIOVISUAL Y CULTURAL (EACEA P9 EURYDICE). (2011). Cifras clave sobre el uso de las TIC para el aprendizaje y la innovación en los centros escolares de Europa 2011. Comisión Europea.

BARRA, E. (2014). Nuevos métodos y herramientas para la creación y utilización de recursos multimedia en la educación. Tesis Doctoral. Valencia: Universidad Politécnica de Madrid.

INSTITUTO DE CIENCIAS DE LA EDUCACION - ÁREA DE SISTEMAS DE INFORMACIÓN Y COMUNICACIONES. (2008). Los objetos de aprendizaje como recurso para la docencia universitaria: criterios para su elaboración. Valencia: Universitat Politècnica de València.

JORDAN, C., PEREZ, M. J., SANABRIA, E. (2014). Investigación del impacto en un aula de matemáticas al utilizar flip education en Pensamiento Matemático, vol. 4, no. 2, p. 9-22.

MAHAJAN G. (2012). "Multimedia in Teacher Education: Perceptions \& Uses" en Journal of Education and Practice, vol. 3, issue 1, p. 5-13.

MOLTÓ, G. (2012) "Producción y Uso de Vídeo-Ejercicios Didácticos en Asignaturas de Programación". En: XVIII Jornadas de Enseñanza Universitaria de la Informática. 255-262.

PRENSKY, M. (2010). Nativos e Inmigrantes Digitales. Cuadernos SEK 2.0.

THE COUNCIL OF EUROPE INTERNET PORTAL, (2013). Bologna for Pedestrians. < http://www.coe.int/t/dg4/highereducation/EHEA2010/BolognaPedestrians_en.asp\#P132_13851> [Consulta: 29 de marzo de 2017]

UNIVERSITAT POLITÈCNICA DE VALÈNCIA (2013). RiuNet, Repositorio Institucional UPV. < https://riunet.upv.es> [Consulta: 29 de marzo de 2017] 